\title{
Temperature-dependent phenotypic variation of Campylobacter jejuni lipooligosaccharides
}

\author{
Evgeny A Semchenko', Christopher J Day', Jennifer C Wilson' ${ }^{1}$ I Darren Grice, Anthony P Moran 1,2, \\ Victoria Korolik ${ }^{1 *}$
}

\begin{abstract}
Background: Campylobacter jejuni is a major bacterial cause of food-borne enteritis, and its lipooligosaccharide (LOS) plays an initiating role in the development of the autoimmune neuropathy, Guillain-Barré syndrome, by induction of anti-neural cross-reactive antibodies through ganglioside molecular mimicry.

Results: Herein we describe the existence and heterogeneity of multiple LOS forms in C. jejuni strains of human and chicken origin grown at $37^{\circ} \mathrm{C}$ and $42^{\circ} \mathrm{C}$, respectively, as determined on sodium dodecyl sulphatepolyacrylamide electrophoresis gels with carbohydrate-specific silver staining and blotting with anti-ganglioside ligands, and confirmed by nuclear magnetic resonance (NMR) spectroscopy. The C. jejuni NCTC 11168 original isolate (11168-O) was compared to its genome-sequenced variant (11168-GS), and both were found to have a lower-M $M_{r}$ LOS form, which was different in size and structure to the previously characterized higher- $M_{r}$ form bearing $G_{1}$ mimicry. The lower- $M_{r}$ form production was found to be dependent on the growth temperature as the production of this form increased from $\sim 5 \%$, observed at $37^{\circ} \mathrm{C}$ to $\sim 35 \%$ at $42^{\circ} \mathrm{C}$. The structure of the lower- $\mathrm{M}_{\mathrm{r}}$ form contained a $\beta$-D-Gal-( $(1 \rightarrow 3)-\beta$-D-GalNAc disaccharide moiety which is consistent with the termini of the $G M_{1}$, asialo- $G M_{1}, G_{1}, G T_{1}$ and $G_{1}$ gangliosides, however, it did not display $G_{1}$ mimicry as assessed in blotting studies but was shown in NMR to resemble asialo-GM . The production of multiple LOS forms and lack of $G_{1} M_{1}$ mimicry was not a result of phase variation in the genes tested of NCTC 11168 and was also observed in most of the human and chicken isolates of $C$. jejuni tested.

Conclusion: The presence of differing amounts of LOS forms at 37 and $42^{\circ} \mathrm{C}$, and the variety of forms observed in different strains, indicate that LOS form variation may play a role in an adaptive mechanism or a stress response of the bacterium during the colonization of different hosts.
\end{abstract}

\section{Background}

Campylobacter jejuni is now well established as the leading cause of bacterial food-borne gastroenteritis worldwide $[1,2]$. Infection symptoms vary in severity and may include nausea, severe or bloody diarrhea, abdominal cramping and fever [3]. C. jejuni infection is usually self-limiting, but in some cases may progress to the debilitating, polyneuropathic disorders Guillain-Barré syndrome (GBS) or the oculomotor variant Miller Fisher syndrome (MFS) $[4,5]$. Importantly, C. jejuni is the commonest antecedent infection in these neuropathies and expression of carbohydrate epitopes mimicking host

\footnotetext{
* Correspondence: v.korolik@griffith.edu.au

'Institute for Glycomics, Griffith University, Gold Coast campus, Queensland, Australia

Full list of author information is available at the end of the article
}

gangliosides is considered a prerequisite for neuropathy development since such mimicry can induce pathogenic, cross-reactive antibodies [6,7]. Gangliosides are glycosphingolipids occurring in high concentration in the peripheral nervous system, particularly in the nerve axon [8]. A humoural response against these glycolipids (e.g. anti- $\mathrm{GM}_{1}, \mathrm{GM}_{1 \mathrm{~b}}, \mathrm{GD}_{1 \mathrm{a}}$, GalNAc- $\mathrm{GD}_{1 \mathrm{a}} \mathrm{GT}_{1 \mathrm{a}}$ and $\mathrm{GQ}_{1 \mathrm{~b}}$ antibodies) plays a central role in GBS and MFS development [6,7]. Mimicry of the saccharide component of gangliosides within the outer core of $C$. jejuni lipooligosaccharides (LOS) is well documented $[9,10]$. Supporting a pathogenic role of C. jejuni in GBS, C. jejuni LOS-induced anti-GM $\mathrm{GM}_{1}$ ganglioside antibodies react at the nodes of Ranvier, where the axon is exposed in the nerve fibre [11], resembling the pathology observed in GBS patients, and inoculation of C. jejuni
C Biomed Central

(c) 2010 Semchenko et al; licensee BioMed Central Ltd. This is an Open Access article distributed under the terms of the Creative Commons Attribution License (http://creativecommons.org/licenses/by/2.0), which permits unrestricted use, distribution, and reproduction in any medium, provided the original work is properly cited. 
$\mathrm{GM}_{1}$-mimicking LOS has been reported to induce GBSlike symptoms in a rabbit model [12].

C. jejuni is capable of growth at temperatures ranging from 30 to $47^{\circ} \mathrm{C}$ and therefore is capable of growth at the body temperatures of human and avian hosts, 37 and $42^{\circ} \mathrm{C}$, respectively $[13,14]$. Different temperature environments may trigger events to accommodate the colonization, commensalism, pathogenesis or dormancy of this bacterium. Over 350 genes have been reported to be differentially expressed at $37^{\circ} \mathrm{C}$ compared to $42^{\circ} \mathrm{C}$, including the galE and wlaE genes found in the LOS biosynthesis locus [15]. Moreover, LOS is an important pathogenic factor of $C$. jejuni. Arising from this, it is possible that $C$. jejuni LOS expression is affected by temperature, whether it is by variable gene expression or at the enzymatic activity level. Although mimicry of gangliosides by $C$. jejuni LOS has been extensively studied structurally over the last two decades $[9,10]$, it is important to note that these previous characterization studies have been performed on strains grown at $37^{\circ} \mathrm{C}$.

The human isolate C. jejuni NCTC 11168 has been a basis for studying this bacterial species since the late 1970s. The sequencing and annotation of its genome was published by the Sanger Centre [16]. A later study revealed that the genome-sequenced strain of $C$. jejuni NCTC 11168 (11168-GS) is a poor colonizer of 1 day-old chicks and showed that this variant had an altered morphology and a different transcriptional profile compared with the original NCTC 11168 isolate (11168-O) [17]. Recurrent passaging of $C$. jejuni $11168-\mathrm{O}$ in laboratory conditions was considered responsible for this variation.

To date, a number of genes from the LOS biosynthesis cluster of C. jejuni NCTC 11168 (HS:2) have been characterized $[4,18]$ and the structures of the lipid $A$ and saccharide components of the LOS have been reported [19-21]. The LOS outer core mimics the oligosaccharide (OS) region of $\mathrm{GM}_{1}$ ganglioside $[20,21]$ and is likely to be capable of switching from a $\mathrm{GM}_{1}$-like epitope to a $\mathrm{GM}_{2}$-like epitope as a result of phase variation [22,23].

The lack of knowledge of the structure of $C$. jejuni LOS at $42^{\circ} \mathrm{C}$ compared to $37^{\circ} \mathrm{C}$ prompted us to examine the effect of incubation temperature on the phenotypic variation of LOS, including the mimicry of gangliosides, in C. jejuni 11168-GS and 11168-O. Variation in LOS structure was assessed by electrophoretic analysis and immunoblotting and confirmed by nuclear magnetic resonance (NMR) spectroscopy. Carbohydrate epitopes produced by both strains were assessed for ganglioside mimicry using various anti-ganglioside ligands (i.e. antibodies, lectins and cholera toxin) as probes. In addition, LOS structural variation at these two incubation temperatures was examined in minimally subcultured $C$. jejuni isolates from humans and chickens. Importantly, notable differences were observed in the relative production by $C$. jejuni of varying size and ganglioside mimicries at $37^{\circ} \mathrm{C}$ and $42^{\circ} \mathrm{C}$.

\section{Results}

\section{Electrophoretic analysis of $C$. jejuni LOS preparations}

Mini-preparations of LOS isolated from $C$. jejuni 11168GS and $11168-\mathrm{O}$ strains grown at $37^{\circ} \mathrm{C}$ and $42^{\circ} \mathrm{C}$ were examined using sodium dodecyl suphate-polyacrylamide gel electrophoresis (SDS-PAGE) analysis. The LOS from C. jejuni $11168-\mathrm{O}$ and 11168-GS strains resolved into two distinct forms, referred to from here on as higher- $\mathrm{M}_{\mathrm{r}}$ and lower- $\mathrm{M}_{\mathrm{r}}$ LOS (Figure 1b). Two control LOS with a known size (Figure 1a) from $M$. catarrhalis serotype A (strain 2951) were used for relative sizing of C. jejuni LOS. The first was wild-type LOS and resolved on the SDS-PAGE with the lower band at $\sim 4 \mathrm{kDa}$ (lane 1). The second was a LOS from a lgt4 mutant $(2951 \Delta l g t 4)$ of $M$. catarrhalis 2951, lacking one glucose and resolved at $\sim 3 \mathrm{kDa}$ [24] (lane 2). Therefore, the difference of one hexose unit corresponded to a relative migration of $\sim 1$ $\mathrm{kDa}$. Accordingly, these controls were used to compare the sizes of the $C$. jejuni LOS forms (Figure $1 \mathrm{~b}$ and 1c). The higher- $\mathrm{M}_{\mathrm{r}}$ form of $C$. jejuni LOS resolved at approximately $6 \mathrm{kDa}$ (and corresponds to the previously described LOS bearing $\mathrm{GM}_{1}$ mimicry [20-23]), whereas the lower- $\mathrm{M}_{\mathrm{r}}$ form, which has not been previously reported, was observed at $\sim 4 \mathrm{kDa}$. Figure $1 \mathrm{~b}$ shows that C. jejuni 11168-O (lanes 3 and 4) and 11168-GS (lanes 5 and 6) have a greater amount of the $4 \mathrm{kDa}$ LOS form at $42^{\circ} \mathrm{C}$, than at $37^{\circ} \mathrm{C}$. For both $11168-\mathrm{O}$ and $-\mathrm{GS}$ at $42^{\circ} \mathrm{C}$ the amount of LOS produced appears greater than at $37^{\circ} \mathrm{C}$, both in terms of quantity of the $6 \mathrm{kDa}$ form and the $4 \mathrm{kDa}$ form. Densitometry analysis revealed that for $11168-\mathrm{O}$ at $37^{\circ} \mathrm{C}$ (Figure $1 \mathrm{~b}$, lane 3 ) $6.3 \%$ of the total LOS produced was the $4 \mathrm{kDa}$ form and $93.7 \%$ was the $6 \mathrm{kDa}$ form. In contrast, at $42^{\circ} \mathrm{C} 35.5 \%$ of total LOS produced was the $4 \mathrm{kDa}$ form and $64.5 \%$ was the $6 \mathrm{kDa}$ form. Similar results were observed for $11168-G S$ variant. These results were confirmed using purified LOS preparations from C. jejuni 11168-O and 11168-GS, which gave identical electrophoretic profiles (data not shown) as those of the LOS mini-preparations. Also, the total amount of protein isolated from the same cell populations of C. jejuni 11168-O and C. jejuni 11168-GS were unaffected by the change of growth temperature (data not shown), thus allowing normalisation of cell samples prior to proteinase $\mathrm{K}$ digestion to produce LOS mini-preparations for comparison. In contrast to LOS, the CPS profiles from the same populations were unaffected by change of growth temperature (data not shown).

The LOS of the wild-type human isolate $C$. jejuni 520 was analysed identically (Figure 1c) to determine whether the temperature-related phenomenon was unique to C. jejuni NCTC 11168. The LOS of strain 520 was found 
(a)

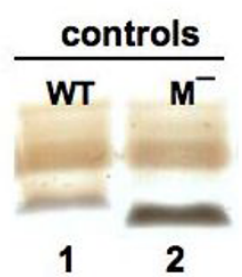

(b)

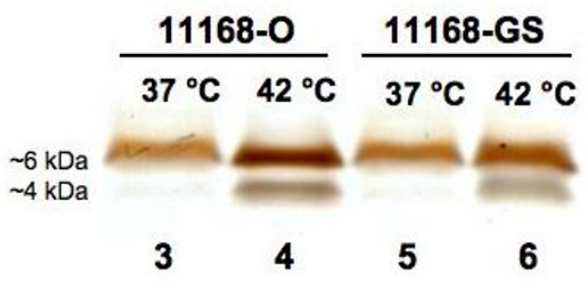

(c)

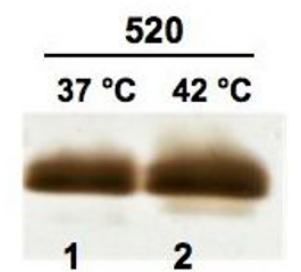

Figure 1 Silver-stained SDS-PAGE gel of the LOS extracted from C. jejuni NCTC 11168 and 520. (a) Controls of M. catarrhalis serotype A (strain 2951) LOS for relative sizing LOS. Lanes: 1, M. catarrhalis wild-type LOS (WT); 2, M. catarrhalis M־mutant LOS lacking the terminal glucose; (b) C. jejuni $11168-\mathrm{O}$ and $11168-\mathrm{GS}$ LOS extracted from bacteria grown at $37^{\circ} \mathrm{C}$ and $42^{\circ} \mathrm{C}$. Lanes: $3,11168-\mathrm{O}$ at $37^{\circ} \mathrm{C} ; 4,11168-\mathrm{O}$ at $42^{\circ} \mathrm{C} ; 5,11168-$ GS at $37^{\circ} \mathrm{C} ; 6,11168-\mathrm{GS}$ at $42^{\circ} \mathrm{C}$. (b) C. jejuni 520 LOS extracts from bacteria grown at $37^{\circ} \mathrm{C}$ and $42^{\circ} \mathrm{C}$. Lanes: 1,520 at $37^{\circ} \mathrm{C} ; 2,520$ at $42^{\circ} \mathrm{C}$. Higher$\mathrm{Mr}_{\mathrm{r}} \mathrm{LOS}$ resolved at $\sim 6 \mathrm{kDa}$ and lower-Mr LOS at $\sim 4 \mathrm{kDa}$.

also to separate into the two distinct forms; the higher- $\mathrm{M}_{\mathrm{r}}$ and lower- $\mathrm{M}_{\mathrm{r}} \mathrm{LOS}$ form. The relative LOS form profile of C. jejuni 520 was also noted to be affected by growth temperature (Figure $1 \mathrm{~b}$ ), whereby a slightly greater amount of the lower- $\mathrm{M}_{\mathrm{r}} \mathrm{LOS}$ was produced at $42^{\circ} \mathrm{C}$ (lane 2).

\section{NMR spectroscopic analysis of the higher- $M_{r}$ and lower- $M_{r}$ LOS form of $C$. jejuni 111168 at $42^{\circ} \mathrm{C}$}

Analysis of the OS isolated from C. jejuni 11168-O at $37^{\circ} \mathrm{C}$ with $1 \mathrm{D}$ NMR gave spectra (data not shown) consistent with the previously published structure of C. jejuni NCTC 11168 [20,21] (Figure 2). Given that the previous structural studies of C. jejuni NCTC 11168 core OS $[20,21]$ had been performed on bacteria grown at $37^{\circ} \mathrm{C}$ it was of interest to investigate the differences in the core OS structure that were observed at $42^{\circ} \mathrm{C}$. To this end, bacteria were grown at $42^{\circ} \mathrm{C}$, the LOS extracted and purified, and the core OS acid-liberated. Examination of the ${ }^{31} \mathrm{P}$ spectrum of the OS so obtained, showed a single ${ }^{31} \mathrm{P}$ peak at $\sim 0 \mathrm{ppm}$, and which was confirmed from a heteronuclear single quantum coherence (HSQC)-total correlation spectroscopy (TOCSY) spectrum to be a phosphorylethanolamine (PEtn) residue. Doubling up of the anomeric line of the signal attributed substitution to the $\rightarrow 3,4,6)$-L- $\alpha$-D-Hep- (C) which is probably due to some heterogeneity in the phosphorylation of the heptose (see Figure 2). Signals consistent with $\alpha$-linked $N$-acetylneuraminic acid
( $\alpha$-Neu5Ac, sialic acid), and $N$-acetylgalactosamine (GalNAc) were also noted. Furthermore, the anomeric region of the HSQC spectrum revealed the presence of nine anomeric signals, in addition to the $\alpha$-Neu5Ac. Taken together, these spectra were consistent with the previously published structure of C. jejuni NCTC 11168 grown at $37^{\circ} \mathrm{C}$ [21] as shown in Figure 2. Nevertheless, examination of the NMR spectra of another isolated minor fraction of the core OS of $11168-\mathrm{O}$ grown at $42^{\circ} \mathrm{C}$ revealed that there was heterogeneity in the fractions with regards to the sialylation of residue $(\mathbf{G})$. Two separate regions of the $1 \mathrm{D}{ }^{1} \mathrm{H}$ are shown in Figure 3; a portion of the anomeric region (5.56-5.70 ppm) and the region of the spectrum where the H3eq protons of $\alpha$ Neu5Ac are expected (2.65-2.85 ppm). Spectrum 3a shows the major fraction consistent with that published in [21]. In spectrum $3 \mathrm{~b}$, the anomeric proton found at $5.67 \mathrm{ppm}$ (residue A) is doubled up and there is a concomitant decrease in the signal intensity of H3eq protons of Neu5Ac. The anomeric resonance of $\mathbf{A}$ is distinct from the other anomeric resonances and conveniently provides a monitor of the structure of the OS in its vicinity. It is expected that the chemical shift of the anomeric resonance of $\mathbf{A}$ would be affected by differences in the sialylation of the galactose $(\mathrm{Gal})$ residue (G). Accordingly, in the minor fraction, which has less sialylation of residue $(\mathbf{G})$, there is the appearance of a new anomeric signal of residue $\mathbf{A}$ at $5.64 \mathrm{ppm}$.

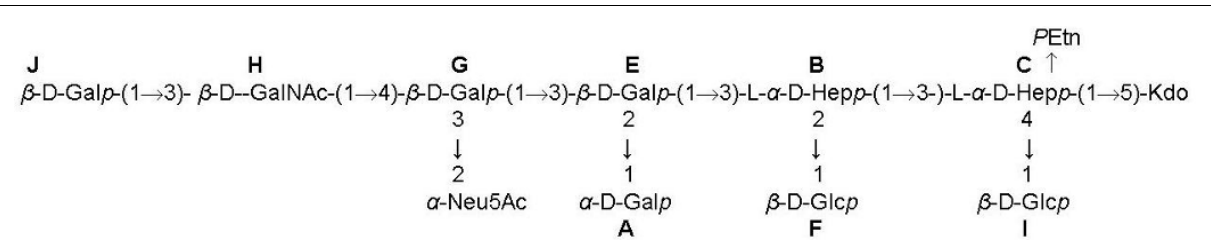

Figure 2 C. jejuni NCTC 11168 core OS structure. Shown is the structure of the higher-M LOS form [20,21], the lower-M form can lack the Neu5Ac residue thereby producing an asialo-GM 1 mimic. Abbreviations: Gal, galactose; GalNAc, N-acetylgalactosamine; Glc, glucose; Hep, heptose; Neu5Ac, N-acetylneuraminic acid Kdo, 3-deoxy-D-manno-oct-2-ulosonic acid; PEtn, phosphorylethanolamine. 

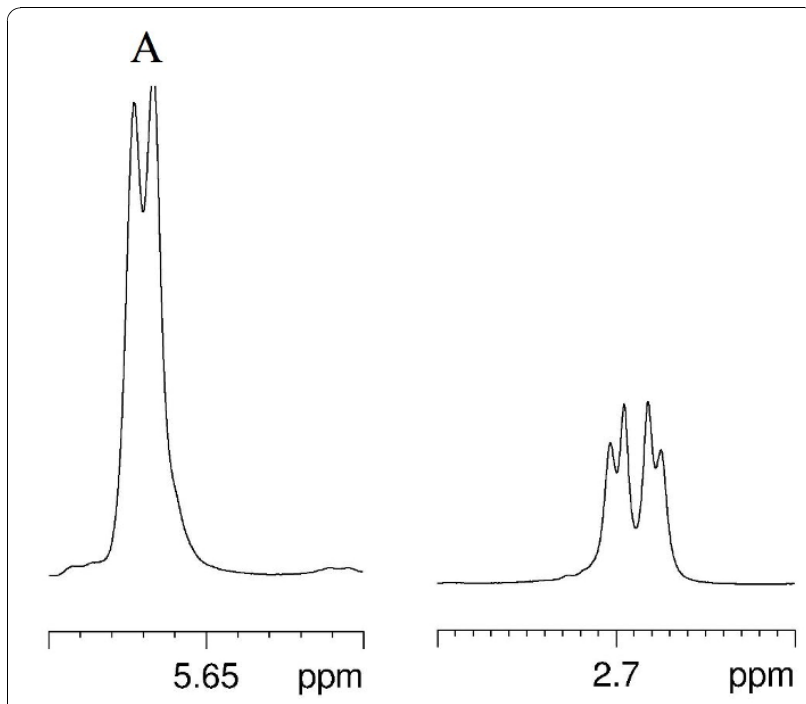

(a)
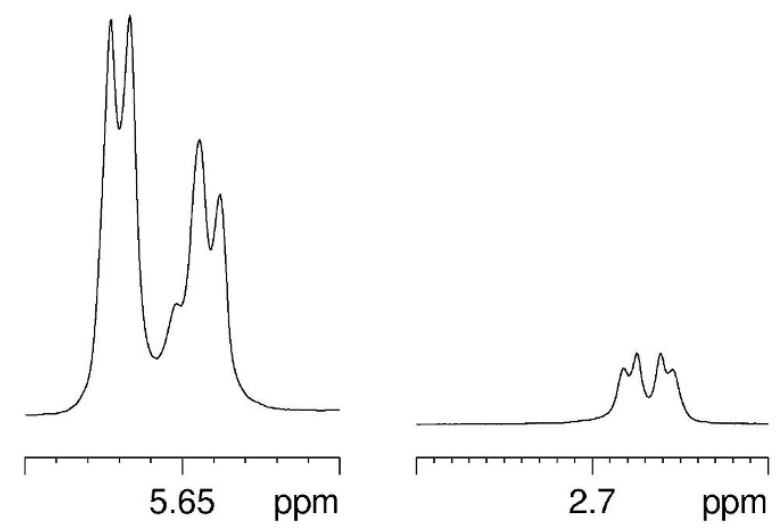

(b)

Figure $3^{1} \mathrm{H}$ 1D spectrum $(298 \mathrm{~K}, 600 \mathrm{MHz})$ of the $C$. jejuni NCTC 11168 OS. (a) The major fraction. (b) The minor fraction. The anomeric signal of residue $\mathbf{A}$ is shown (between $5.62-5.70 \mathrm{ppm}$ ) and the H3eq proton of $\alpha$-Neu5Ac (between 2.65-2.85 ppm).

Collectively, the NMR data shows that there is a difference in sialylation between the higher- $\mathrm{M}_{\mathrm{r}}$ form of $C$. jejuni $11168 \mathrm{LOS}(\sim 6 \mathrm{kDa})$ and the lower- $\mathrm{M}_{\mathrm{r}}$ form $(\sim 4 \mathrm{kDa})$; in the latter Neu5Ac can be absent, thus exhibiting asialo$\mathrm{GM}_{1}$ mimicry. Sialic acid is a 9-carbon sugar and has different charge properties to hexose sugars, which accounts for the approximately $2 \mathrm{kDa}$ difference in apparent mass of the two LOS forms as seen in Figure 1.

\section{Analysis of $\mathrm{GM}_{1}$ epitope mimicry in C. jejuni LOS using cholera toxin subunit $B$ (CTB)}

C. jejuni 11168-GS has been previously reported to mimic the structure of the $\mathrm{GM}_{1}$ ganglioside and hence displays strong binding to CTB [20-23,25]. Therefore, to determine whether the higher- or lower- $\mathrm{M}_{\mathrm{r}}$ LOS forms of $C$. jejuni $11168-\mathrm{O}$ and 11168-GS mimic the $\mathrm{GM}_{1}$ epitope, the ability of both LOS forms to bind CTB was analysed using a blotting assay. The higher- $\mathrm{M}_{\mathrm{r}} \mathrm{LOS}$ of C. jejuni $11168-\mathrm{O}$ and $11168-\mathrm{GS}$ isolates grown at $37^{\circ} \mathrm{C}$ or $42^{\circ} \mathrm{C}$ bound CTB strongly (Figure 4 , lanes $1-4$ ). On the other hand, the lower- $\mathrm{M}_{\mathrm{r}}$ LOS did not bind to CTB, indicating that it does not exhibit $\mathrm{GM}_{1}$ mimicry. In contrast, the higher- $\mathrm{M}_{\mathrm{r}}$ LOS form of $C$. jejuni strain 520 grown at $37^{\circ} \mathrm{C}$ or $42^{\circ} \mathrm{C}$ bound $\mathrm{CTB}$ weakly, indicating that the saccharide terminus may exhibit some ganglioside-related mimicry, though probably not $\mathrm{GM}_{1}$. Binding of CTB to the lower- $\mathrm{M}_{\mathrm{r}}$ form was not detected (Figure 4 , lanes 5 and 6).

\section{Analysis of $C$. jejuni LOS epitope mimicry using peanut agglutinin (PNA)}

To further investigate the molecular mimicry of C. jejuni 11168 LOS forms, lectin blotting was performed using PNA which binds $\beta$-D-Gal-( $1 \rightarrow 3)$-D-GalNAc and $\beta$-D-Gal $(1 \rightarrow 3)$-D-Gal. The disaccharide $\beta$-DGal-(1 $\rightarrow 3)-D-G a l N A c$ is present as the terminal disaccharide of $\mathrm{GM}_{1}$ ganglioside, but is also present in other gangliosides (e.g. asialo-GM1, $\mathrm{GD}_{1}, \mathrm{GT}_{1}$ and $\mathrm{GQ}_{1}$ gangliosides). PNA strongly bound both the higher- $\mathrm{M}_{\mathrm{r}}$ and lower- $\mathrm{M}_{\mathrm{r}}$ LOS forms of C. jejuni 11168-O and 11168GS grown at 37 and $42^{\circ} \mathrm{C}$ (Figure 5, lanes 1-4). Binding of the PNA to the higher- $\mathrm{M}_{\mathrm{r}}$ LOS is consistent with the presence of $\mathrm{GM}_{1}$-like mimicry and $\mathrm{CTB}$ binding observed above. Binding of PNA to the lower- $\mathrm{M}_{\mathrm{r}} \mathrm{LOS}$ is also probably due to the occurrence of a terminal $\beta$-DGal-(1 $\rightarrow 3)$-D-GalNAc in the truncated lower- $\mathrm{M}_{\mathrm{r}}$ LOS. Taking the results of CTB and PNA together suggests that the most likely structure for the lower- $\mathrm{M}_{\mathrm{r}}$ LOS form is an asialo- $\mathrm{GM}_{1}$-like structure.

In contrast, both higher- $\mathrm{M}_{\mathrm{r}}$ and lower- $\mathrm{M}_{\mathrm{r}}$ LOS of C. jejuni 520 did not bind PNA (Figure 5; lanes 5-6) in

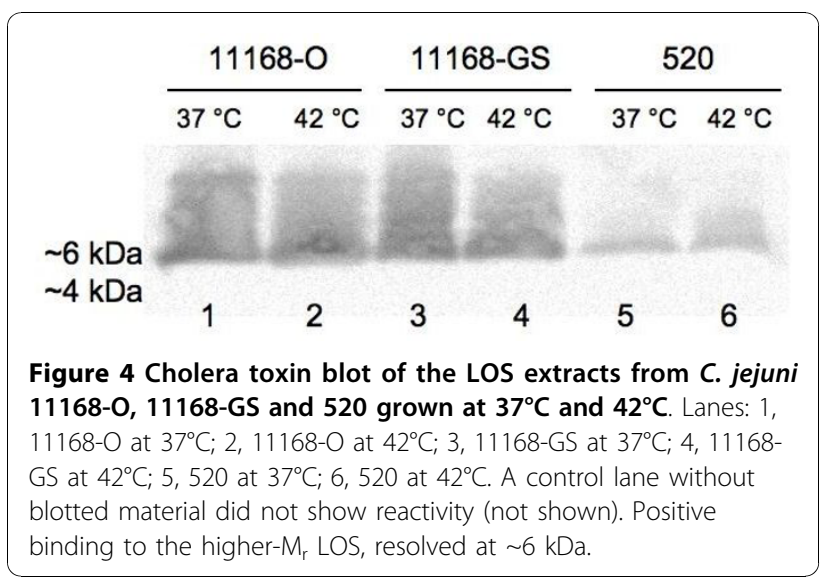




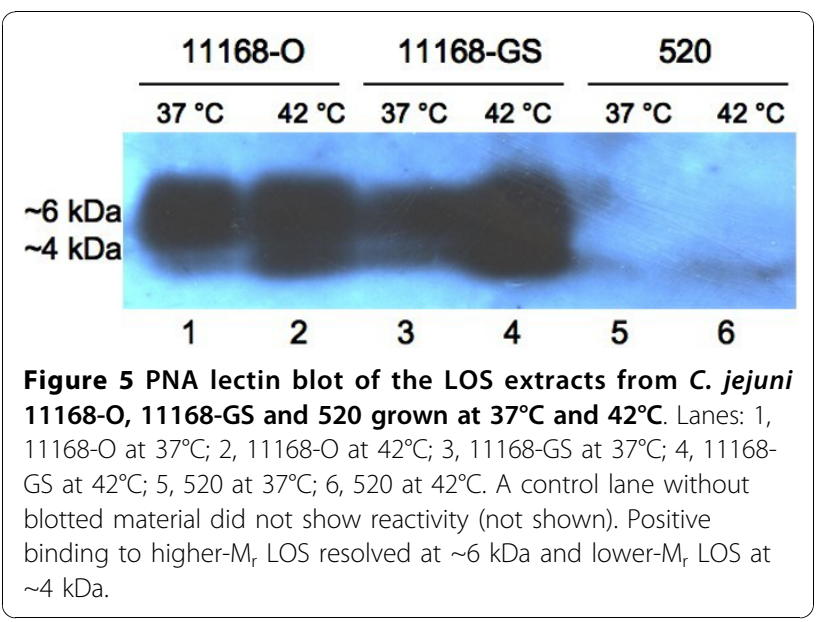

a similar blotting procedure. This finding was consistent with the results of CTB-binding analysis of the LOS with this strain and indicated the absence of $\mathrm{GM}_{1}$-like mimicry, but does not exclude other ganglioside mimicry in the LOS forms of C. jejuni 520.

\section{Analysis of LOS from C. jejuni NCTC 11168-O single colonies}

To determine whether the production of multiple LOS forms occurs as a result of a phase variation, LOS minipreparations from 30 randomly selected, single colonies of C. jejuni $11168-\mathrm{O}$ grown at 37 or $42^{\circ} \mathrm{C}$ were analysed. Higher- and lower- $M_{r}$ LOS forms were present within each clonal population of $C$. jejuni 11168-O grown at 37 or $42^{\circ} \mathrm{C}$. Figure 6 shows a representative sample of LOS profiles from single colonies grown at $42^{\circ} \mathrm{C}$ which showed identical profiles with $\sim 35.5 \%$ of the total LOS produced being of $4 \mathrm{kDa}$ form and $\sim 64.5 \%$ of the $6 \mathrm{kDa}$ form. LOS profiles for single C. jejuni 11168-O colonies grown at $37^{\circ} \mathrm{C}$ were also identical to each other and to that shown in Figure 1b, lane 3 (data not shown). Equally strong binding of CTB to higher- $\mathrm{M}_{\mathrm{r}} \mathrm{LOS}$ was

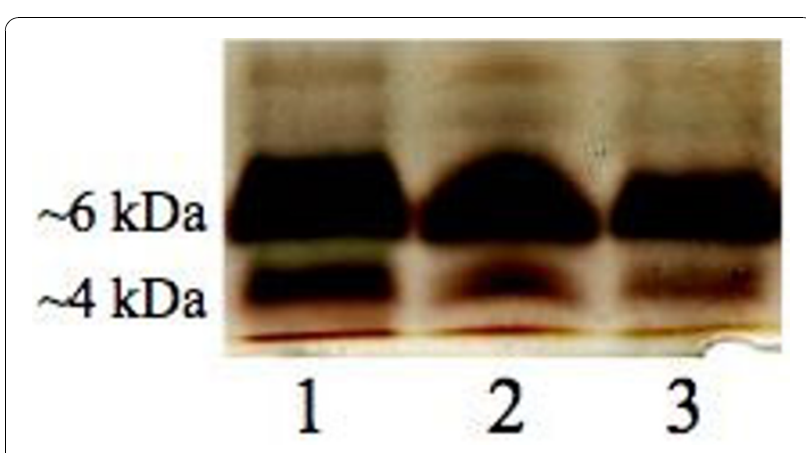

Figure 6 Silver-stained SDS-PAGE gel of LOS extracted from single colonies of $C$. jejuni $11168-0$ grown at $42^{\circ} \mathrm{C}$. Lanes: $1-3$, LOS from selected individual colonies. Higher- $\mathrm{M}_{\mathrm{r}}$ resolved at $\sim 6 \mathrm{kDa}$ and lower-M $\mathrm{M}_{\mathrm{r}} \mathrm{LOS}$ observed at $\sim 4 \mathrm{kDa}$. observed for all the colonies tested suggesting that the phenomenon is unlikely to have been caused by phase variation. This was further confirmed by DNA sequence analysis of homopolymeric G- and A-tracts in wlaN and cj1144-45c genes as described below.

The observed $\mathrm{GM}_{1}$ mimicry of the LOS epitope was also confirmed by a colony lift whereby each of the single colonies of $C$. jejuni $11168-\mathrm{O}$ grown at $37^{\circ} \mathrm{C}$ was found to bind the $\mathrm{GM}_{1}$-binding ligand CTB (data not shown).

\section{Analysis of the homopolymeric tracts from the phase variable genes wlaN and cj1144-45c in C. jejuni NCTC 11168-O single colonies}

To further examine the nature of LOS variation in C. jejuni, gene expression of the homopolymeric regions of two known phase variable genes, wlaN (responsible for addition of terminal Gal to OS [23]) and cj1144-45c (function unknown), located in the LOS biosynthesis locus of $C$. jejuni were analysed. Both genes were amplified from 20 randomly selected single colonies of C. jejuni $11168-\mathrm{O}$ grown at $42^{\circ} \mathrm{C}$ and were subsequently sequenced. Each clonal population contained an 8-residue G-tract in the wlaN, which allows for complete translation of the gene. The sequence of $c 1144-45 c$ was consistently found to contain a 9-residue G-tract which interrupts the reading frame. In addition, a homopolymeric A-tract of $c j 1144-45 c$ was also examined and no sequence variation could be detected in any of the clonal populations. As further confirmation of the lack of phase variation in the wlaN and $c j 1144-45 c$ genes, the total bacterial cell population from a confluent agar plate, was subjected to similar polymerase chain reaction (PCR) analysis and sequence analysis and consistently only a single sequence for each homopolymeric tract was detected. These analyses confirmed that the growth temperature did not induce sequence variation in the lengths of the homopolymeric G-tract and A-tract in cj1144-45c as well as in the G-tract of wlaN of $C$. jejuni 11168-O.

\section{LOS form variation in human and chicken isolates of C. jejuni}

C. jejuni strains originally isolated from human patients and broiler chickens were examined to determine whether multiple LOS forms are common in Campylobacter strains (Table 1). Figure 7a illustrates the diversity of the LOS forms observed in extracts from a representative selection of human and chicken isolates of C. jejuni from those listed in Table 1. C. jejuni chicken isolates strains 331, 434, 506, 7-1 and RM1221 expressed both higher and lower- $\mathrm{M}_{\mathrm{r}}$ LOS forms whereas in strains 913, 019 and 008 only the higher- $M_{r}$ LOS form was detected (Table 1). All the human isolates 
Table 1 Summary of the LOS phenotypes from different C. jejuni isolates

\begin{tabular}{|c|c|c|c|}
\hline Origin & $\begin{array}{l}\text { C. jejuni } \\
\text { strain }\end{array}$ & $\begin{array}{c}\text { LOS phenotype at } \\
37^{\circ} \mathrm{C}\end{array}$ & $\begin{array}{c}\text { LOS phenotype at } \\
42^{\circ} \mathrm{C}\end{array}$ \\
\hline \multirow[t]{8}{*}{ chicken } & $331^{*}$ & $\mathrm{H}, \mathrm{L}$ & $H, L$. \\
\hline & 434 & $H, L$ & $H, L$. \\
\hline & $506^{*}$ & $H, L$ & $H, L$ \\
\hline & $913^{*}$ & $\mathrm{H}$ & $\mathrm{H}$ \\
\hline & 008 & $\mathrm{H}$ & $\mathrm{H}$ \\
\hline & 019 & $\mathrm{H}$ & $\mathrm{H}$ \\
\hline & 7-1 & $\mathrm{H}, \mathrm{L}$ & $\mathrm{H}, \mathrm{L}$ \\
\hline & RM1221 & $H, L$ & $\mathrm{H}, \mathrm{L}$ \\
\hline \multirow[t]{9}{*}{ human } & $224^{*}$ & $\mathrm{H}, \mathrm{L}$ & $H, L \cdot$ \\
\hline & 291 & $\mathrm{H}, \mathrm{L}$ & $H, L$ \\
\hline & 351 & $H, L$ & $H, L$ \\
\hline & 375 & $\mathrm{H}$ & $\mathrm{H}$ \\
\hline & 388 & $H, L$ & $H, L$ \\
\hline & $421^{*}$ & $H, L$ & $H, L$. \\
\hline & 520 & $\mathrm{H}, \mathrm{L}$ & $\mathrm{H}, \mathrm{L}$ \\
\hline & $11168-G S^{*}$ & $H, L$ & $H, L \cdot$ \\
\hline & 81116 & $\mathrm{H}, \mathrm{L}$ & $\mathrm{H}, \mathrm{L}$ \\
\hline
\end{tabular}

$\mathrm{H}$, higher-M $\mathrm{M}_{\mathrm{r}}$ LOS form.

$L$, lower-Mr $\mathrm{LOS}$ form.

- Increase in the amount of LOS produced.

*Shown in Figure 7. were found to express both higher- and lower- $\mathrm{M}_{\mathrm{r}} \mathrm{LOS}$ forms except for strain 375 where only one $M_{r}$ form (higher- $\mathrm{M}_{\mathrm{r}}$ form) was detected (Table 1). C. jejuni strains 331 (chicken), 434 (chicken), 224 (human), 421 (human) and 11168 (human) were also shown to increase the production of lower- $\mathrm{M}_{\mathrm{r}} \mathrm{LOS}$ form, and a corresponding total increase in LOS production, at $42^{\circ} \mathrm{C}$ in contrast to $37^{\circ} \mathrm{C}$ (Table 1 ).

A CTB blot of LOS from a representative selection of human and chicken isolates of C. jejuni (Figure 7b), demonstrated the variability in LOS expression in different strains with respect to ganglioside mimicry. Only the higher- $M_{r}$ LOS form was found to bind CTB in the tested strains. Furthermore, the higher- $\mathrm{M}_{\mathrm{r}} \mathrm{LOS}$ of some C. jejuni strains (506 and 913) did not bind CTB, indicating the absence of $\mathrm{GM}_{1}$ ganglioside mimicry in both forms of LOS.

\section{Discussion}

This study has shown that $C$. jejuni NCTC 11168-O and 11168-GS, as well as most randomly chosen chicken and human strains produce at least two distinct LOS forms when incubated at the core temperatures of human $\left(37^{\circ} \mathrm{C}\right)$ and avian $\left(42^{\circ} \mathrm{C}\right)$ hosts. This is consistent with previous observations that $C$. jejuni is capable of producing a variety

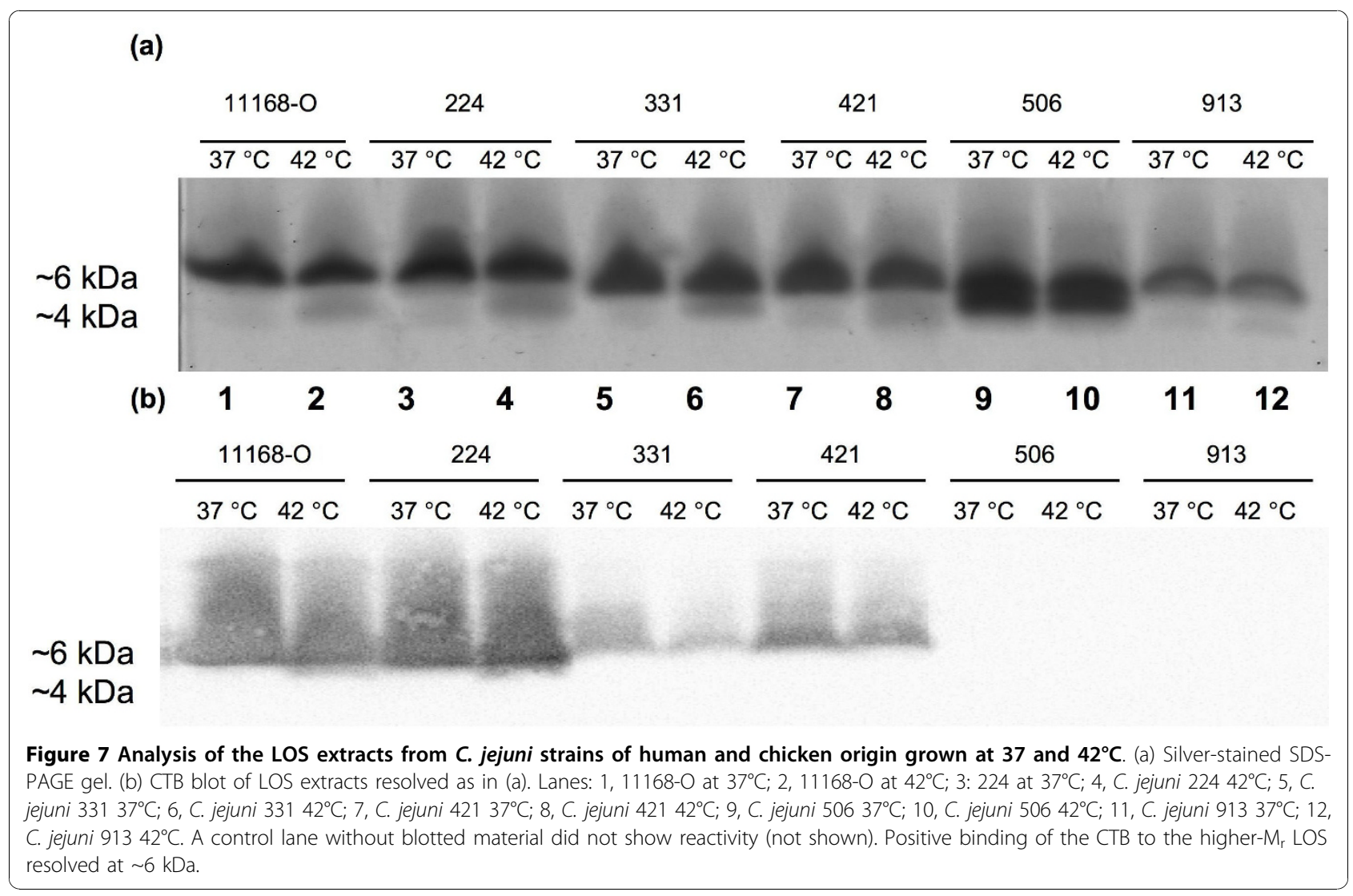


of polysaccharide-related structures that are influenced by growth conditions, such as temperature [26].

Surface antigen modulation and generation of hostadapted variants are common attributes of many bacteria and enhance the pathogenicity and survivability of the microorganism, as well as the ability to evade the host immune response during the infection [27]. This variation may be achieved through several mechanisms, such as differential gene expression or enzymatic activity and specificity modulation, which can be triggered by a random and/or environmental stimuli [28]. It is possible to speculate that in the case of C. jejuni LOS, glycosyl transferases have the highest activity or are more stable promoting maximum functionality.

It is interesting to note that the growth temperature of C. jejuni NCTC 11168 was previously reported to influence the oxidative stress response [14]. In addition, approximately $20 \%$ of $C$. jejuni genes were reported to be up- or down-regulated in response to increasing the temperature from 37 to $42^{\circ} \mathrm{C}$, including genes from the LOS and protein glycosylation clusters [15]. However, the change in LOS phenotype was not resolved to date.

In the present study, the phenotypic expression of the lower- $\mathrm{M}_{\mathrm{r}}$ LOS form appeared to be modulated by the growth temperature. This prompts speculation that the increased production of this form may be directly or indirectly caused by either specific changes in gene expression of the glycosyltransferases or glycosyl synthesis genes affecting LOS biosynthesis, thus leading to the production of varying amounts of the LOS forms. Due to the apparent loss of sialylation in the lower $M_{r}$ LOS structure it is likely that the variation of the structure is attributable to functional differences in the synthesis of the transport machinery of sialic acid under the different temperatures. We consider the most likely candidate for this difference to be the dual functioning enzyme, GalNAc transferase and CMP-Neu5Ac synthase, CgtA [18].

It is also tempting to speculate that the increased production of the lower- $\mathrm{M}_{\mathrm{r}} \mathrm{LOS}$ form at $42^{\circ} \mathrm{C}$ might play a role in the bacterial-host interactions of $C$. jejuni. The increased production of the $4 \mathrm{kDa}$ form which occurred at $42^{\circ} \mathrm{C}$, the avian host body temperature, raises a possibility that this form could contribute to the commensalism by this bacterium in poultry [17]. The increase at $37^{\circ} \mathrm{C}$ in the proportion of the higher $\mathrm{M}_{\mathrm{r}} \mathrm{LOS}$, the portion of the LOS that is sialylated and is a $\mathrm{GM}_{1}$ mimic $[20,21]$, indicates an increase in the production of an LOS structure that is thought to have a role in immune evasion and survival in mammalian hosts [29]. These hypotheses, however, will require further investigation, particularly chicken and murine infection studies.

Phase variation is the most commonly described mechanism, for antigenic variation and changes in the phenotype of the microorganism. Like Neisseria meningitidis and Haemophilus influenzae, C. jejuni is also known to exhibit modulation of its surface polysaccharide structures as a result of phase variation [27,30]; however, this does not appear to be the case with production of the temperature-related LOS form in C. jejuni. Both forms were consistently produced by all clonal populations of C. jejuni 11168-O examined in this study suggesting that modulation of LOS forms is unlikely to be caused by phase variation. Furthermore, we have analyzed the "on-off" status of phase variable genes (wlaN and cj1144-45c) in C. jejuni LOS biosynthesis cluster to further demonstrate that the described variation of LOS forms is not being caused by phase variation of LOS genes. C. jejuni $11168-\mathrm{O}$ grown at $42^{\circ}$ $\mathrm{C}$ was used in this experiment as it shows greater abundance of the lower- $\mathrm{M}_{\mathrm{r}}$ LOS form, hence increasing the chance of detecting changes in phase variable genes. Lengths of the homopolymeric $\mathrm{G}$ and A tracts from wlaN and $c j 1144-45 c$ genes did not vary in any of 20 randomly selected colonies, suggesting that these genes are under regulatory mechanisms unaffected by growth temperature and the described variation of LOS forms is not caused by variation in the lengths of the homopolymeric tracts. Furthermore, no change in the $\mathrm{GM}_{1}$ mimicry of the clonal populations had been observed. It is also interesting to note that not all strains of $C$. jejuni expressed multiple LOS forms irrespective of the isolation origin, human or chicken.

No differences were observed in the production of the various LOS forms between the two variants of 11168 , the genome sequenced and original isolate. The higher$\mathrm{M}_{\mathrm{r}}$ form of C. jejuni 11168 ( $\left.6 \mathrm{kDa}\right)$ exhibited $\mathrm{GM}_{1}$-like mimicry and, therefore, corresponded to the previously characterized LOS $[20,21,23]$. Studies with CTB, a wellknown binder of $\mathrm{GM}_{1}$ ganglisoide [25], confirmed the presence of a $\mathrm{GM}_{1}$ mimic in this form of NCTC 11168. Similar mimicry was also detected among the higher- $\mathrm{M}_{\mathrm{r}}$ LOS forms of the other isolates of humans and chickens tested, but not in the lower- $\mathrm{M}_{\mathrm{r}}$ form of any other strains. The weak binding of CTB to the higher-M $\mathrm{M}_{\mathrm{r}}$ LOS variant of $C$. jejuni 520 reflects that the saccharide terminus may exhibit some ganglioside-related mimic, though not $\mathrm{GM}_{1}$ mimicry. This is shown by the CTB binding to ganglioside-related structures not just $\mathrm{GM}_{1}$ and PNA did not confirm the presence of a terminal $\beta$-D-Gal-( $1 \rightarrow 3)$-D-GalNAc. A CTB binding affinity study showed that the lower- $\mathrm{M}_{\mathrm{r}}$ form of $C$. jejuni NCTC 11168 failed to bind to the lectin. Nevertheless, the results of the present study showed that it contains a $\beta$-D-Gal-(1 $\rightarrow 3)$-D-GalNAc disaccharide moiety in the core consistent with production of a truncated (because of its lower molecular mass), but related form, of the NCTC 11168 structure previously described [21], and is an asialo- $\mathrm{GM}_{1}$-like structure. 


\section{Conclusion}

In conclusion, this study identified the presence of a lower- $\mathrm{M}_{\mathrm{r}}$ LOS form produced by C. jejuni NCTC 11168 and other clinical and avian strains. The lower- $\mathrm{M}_{\mathrm{r}}$ form production was growth-temperature related as higher quantities were observed at $42^{\circ} \mathrm{C}$. It is tempting to speculate that the occurrence of greater quantities of this form at avian body temperature might play a role in an adaptative mechanism to aid commensal colonization of such hosts. Alternatively, changes in the relative production of the two forms of LOS at the higher temperature could be related to a stress response. Such a phenomenon has already been seen with increased oxygen tension in the growth atmosphere of $C$. jejuni influencing the structural mimicry exhibited in the LOS of this bacterium [31]. Although an intriguing phenomenon, further investigations are required to evaluate these alternate hypotheses.

\section{Methods}

\section{Bacterial strains and growth conditions}

The original isolate of C. jejuni NCTC 11168 (11168-O) that had been characterized by Gaynor et al. (2004) [17], C. jejuni 11168-GS (genome-sequenced NCTC 11168) that had been sequenced and annotated at the Sanger Centre (Hinxton, Cambridge, UK) [16], and strain 81116 were kindly supplied by D.J. Newell (Veterinary Laboratories Agency, Weybridge, UK). C. jejuni RM1221 has been described [32] and was kindly provided by R. E. Mandrell (United States Department of Agriculture, CA, USA.). C. jejuni clinical isolates 224, $291,351,375,388,421,520$, and chicken isolates 008 , $019,331,434,506,913,7-1$ were obtained from the Royal Melbourne Institute of Technology (Melbourne, Vic., Australia) and Griffith University (Gold Coast, Qld., Australia) culture collections. All C. jejuni strains were subcultured no more than once to avoid the influence of passaging. Strains were grown on blood agar, composed of Columbia agar containing $5 \%(\mathrm{v} / \mathrm{v})$ defibrinated horse blood and Skirrow's antibiotic supplement (Oxoid), under microaerobic conditions $\left(5 \% \mathrm{O}_{2}, 10 \%\right.$ $\mathrm{CO}_{2}$ and $85 \% \mathrm{~N}_{2}$ ) at $37^{\circ} \mathrm{C}$ for $48 \mathrm{~h}$ and $42^{\circ} \mathrm{C}$ for $24 \mathrm{~h}$.

\section{LOS preparations}

\section{For gel electrophoresis}

Blood agar-grown bacteria were harvested in $1 \mathrm{~mL}$ of sterile water, washed once in $1 \mathrm{~mL}$ of sterile water, and lysed by heating. Prior to lysis, samples were adjusted for numbers of bacteria using the $\mathrm{OD}_{600}$ measurements of bacterial suspensions. Mini-preparations of LOS were prepared by treating the whole-cell extracts with proteinase $\mathrm{K}$ as described previously [33]. The LOS mini-preparations from single colonies were prepared by collecting and washing cells in $40 \mu \mathrm{L}$ of sterile water and then lysing by heating. Purified $C$. jejuni LOS was prepared by subjecting the biomass to hot phenol-water treatment using $90 \%(\mathrm{v} / \mathrm{v})$ aqueous phenol at $65^{\circ} \mathrm{C}$ for 10 min [34]. Extracted LOS was purified by enzymatic treatment as described previously [19]. The LOS preparations were made up to $15 \mu \mathrm{g} / \mu \mathrm{L}$ in distilled water prior to gel electrophoresis.

\section{For NMR analysis}

C. jejuni 11168 was grown for $24 \mathrm{hr}$ as described above and bacterial biomass was harvested and washed twice using phosphate-buffered saline pH 7.4 (PBS; Sigma) and centrifugation $\left(5000 \times g, 4^{\circ} \mathrm{C}, 15 \mathrm{~min}\right)$. Biomass was lyophilised and $21 \mathrm{~g}$ and $20 \mathrm{~g}$ dry-cell mass was collected from cultures grown at $37^{\circ} \mathrm{C}$ and $42^{\circ} \mathrm{C}$, respectively. Dried biomass was pretreated using pronase-E [35]. Extraction of LOS was carried out using hot-phenol water technique [34]. Water-soluble LOS was purified using RNaseA, DNase II and proteinase K (Sigma) and ultra-centrifugation, as previously described [19]. The LOS were treated with $0.1 \mathrm{M} \mathrm{HCl}$ at $100^{\circ} \mathrm{C}$ for 2 hours to cleave the acid-labile ketosidic linkage between the core OS and lipid A [19]. The lipid A precipitate was removed by centrifugation $\left(5000 \times g, 4^{\circ} \mathrm{C}, 30 \mathrm{mins}\right)$, washed and both this and supernatant were lyophilised. The supernatant was fractionated using gel-permeation chromatography on a column of Bio-Gel P4 $(1 \mathrm{~m} \times 2 \mathrm{~cm})$ with 0.05 $\mathrm{M}$ pyridinium acetate ( $\mathrm{pH} 4.5$ ) as the eluent. The resultant fractions were monitored by capillary-tube spotting on silica gel 60 TLC plates (Merck), followed by charring with $20 \% \mathrm{H}_{2} \mathrm{SO}_{4}$ in $\mathrm{EtOH}$ at $150^{\circ} \mathrm{C}$. The water-soluble carbohydrate-containing fractions of core OS were flashfrozen in dry-ice/acetone bath and lyophilized.

\section{CPS and whole-cell protein preparations}

For assessing CPS production, proteinase K-treated whole cell extracts were prepared as described above. Whole-cell protein samples were prepared by incubating SDS-PAGE loading buffer with C. jejuni biomass at $100^{\circ}$ $\mathrm{C}$ for $5 \mathrm{~min}$ to facilitate bacterial lysis and binding of the SDS to the denatured proteins.

\section{Electrophoretic analyses}

Equal quantities of samples, either LOS mini-preparations or purified LOS, and CPS samples were resolved on $10 \%$ (v/v) SDS-PAGE containing urea $(6 \mathrm{M})$ and tricine $(0.3$ $\mathrm{mM})$ (Tricine-SDS-PAGE) with tricine-containing cathode buffer as previously described [36]. Stacking and separating gels contained $5.5 \%$ and $10 \%(\mathrm{v} / \mathrm{v})$ acrylamide, respectively. Following the electrophoresis of LOS samples, gels were fixed and the resolved molecules were detected using the carbohydrate silver staining method [37] or CPS by Alcian Blue staining [38]. Electrophoresis was conducted at $30 \mathrm{~V}$ for $1 \mathrm{~h}$ to maximize stacking and then separated at 
$200 \mathrm{~V}$ for $30 \mathrm{~min}$. Whole-cell protein samples were resolved on glycine-buffered $15 \%(\mathrm{v} / \mathrm{v})$ polyacrylamide gels (Glycine-SDS-PAGE) as previously described [39]. Electrophoresis was conducted at $100 \mathrm{~V}$ for $1.5 \mathrm{~h}$. Proteins were detected by conventional Coomassie Blue staining [19]. Densitometry image analysis was performed using the QuantityOne software package (BioRad). The published $M$. catarrhalis LOS from M. catarrhalis wild-type (strain 2951) and the lgt4 LOS biosynthesis mutant [24] were used as a control for relative size determination of LOS structures due to the loss of a single hexose sugar from the known OS structure.

\section{NMR spectroscopy}

Purified OSs were dissolved in $\mathrm{D}_{2} \mathrm{O}$ (CIL 99.998\%) and cycled through 3 steps of lyophilization/dissolution to remove exchangeable protons. ${ }^{1} \mathrm{H}$ and ${ }^{13} \mathrm{C}$ NMR experiments were performed at $600 \mathrm{MHz}$ and 150 $\mathrm{MHz}$ respectively at $298 \mathrm{~K}$ or $278 \mathrm{~K}$ in $\mathrm{D}_{2} \mathrm{O}$ using a Bruker Avance spectrometer. Chemical shifts are reported in ppm referenced to DSS. Spectral assignment was aided by recording of ${ }^{1} \mathrm{H} 1 \mathrm{D}$, gradient correlation spectroscopy (COSY), TOCSY, (60 and $120 \mathrm{~ms}$ mixing time), ${ }^{13} \mathrm{C}$ attached proton test (APT), ${ }^{1} \mathrm{H}_{-}{ }^{13} \mathrm{C}$ HSQC and edited ${ }^{1} \mathrm{H}_{-}{ }^{13} \mathrm{C}-\mathrm{HSQC}\left(\mathrm{CH}\right.$ and $\mathrm{CH}_{2}$ correlations opposite sign), ${ }^{1} \mathrm{H}_{-}{ }^{13} \mathrm{C}-\mathrm{HSQC}$-TOCSY and edited ${ }^{1} \mathrm{H}_{-}{ }^{13} \mathrm{C}$-HSQC-TOCSY (60 and $120 \mathrm{~ms}$ mixing time) (one bond $\mathrm{C}-\mathrm{H}$ correlations opposite sign), and ${ }^{1} \mathrm{H}_{-}{ }^{13} \mathrm{C}-\mathrm{HSQC}$-nuclear Overhauser enhancer spectroscopy (-NOESY), NOESY (400 ms) spectra. In addition, 1D selective TOCSY experiments were used to assist with the assignment process. All spectra were acquired using unmodified pulse sequences from the Bruker pulse sequence library.

\section{Ligand and Western blotting}

In addition to chemical staining, the fractionated $C$. jejuni LOS was transferred from Tricine SDS-PAGE gels onto a Pall ${ }^{\circ}$ PVDF membrane using a semi-dry transblotter (Bio-Rad). After transfer, the membrane was reacted with horseradish peroxidase-(HRP-) conjugated CTB $\left(3 \mu \mathrm{g} \mathrm{mL}{ }^{-1}\right)$, or with HRP-conjugated PNA (lectin from Arachis hypogaea) $\left(5 \mu \mathrm{g} \mathrm{mL^{-1 }}\right)$, or with HRPconjugated anti-GM $\mathrm{GM}_{1}$ ganglioside IgG (diluted 1:3000) in PBS. Membranes were developed using HRP Color Development Solution (Bio-Rad) or SuperSignal HRP Chemiluminescent Substrate (Thermo Scientific) according to the manufacturer's instructions.

\section{Colony lift}

C. jejuni was grown on Columbia agar containing $2 \%$ $(\mathrm{v} / \mathrm{v})$ horse blood under microaerobic conditions as described above. The lower concentration of blood was used to reduce the background during the blotting procedure. Colonies were bound to the nitrocellulose membrane by overlaying the agar plate. The membrane was then baked for $1 \mathrm{~h}$ at $80^{\circ} \mathrm{C}$ as described elsewhere [40]. Subsequently, the membrane was blotted with HRPCTB as described above.

\section{Amplification and sequencing of phase variable genes wlaN and cj1144-45c}

For PCR wlaN G-tract forward (GATATAGCTAAAGA GTATGCTAGTAAAG) wlaN G-tract reverse (GGATAATATAATAAGGCATCTTCTGCC) and $c j 1144-45 c$ G-tract forward (GGGTTGATGAAGCAAGAAATTAGTAG) $c j 1144-45 c$ G-tract reverse (GCTAAAAACCA AGGTCCTATAACACC) primer combinations wee used. Twenty (20) reactions were inoculated with bacteria from single colonies of $C$. jejuni 11168-O grown at $42^{\circ} \mathrm{C}$. Amplified $\sim 500 \mathrm{bp}$ fragments were cleaned up using an Eppendrof Perfectprep Gel Cleanup kit and were sent for sequencing at the Australian Genome Research Facility (University of Queensland, St. Lucia, Brisbane, QLD, Australia).

\section{Abbreviations}

CPS: capsular polysaccharide; CTB: cholera toxin subunit B; COSY: correlation spectroscopy; HRP: horseradish peroxidase; HSQC: heteronuclear single quantum coherence; GBS: Guillain-Barré syndrome; Gal: galactose; GalNAc: galactosamine; LOS: lipooligosaccharide; $M_{r}$ : molecular mass; MFS: Miller Fisher syndrome; Neu5Ac sialic acid: (N-acetylneuraminic acid); NMR: nuclear magnetic resonance; OS: oligosaccharide; PBS: phosphate-buffered saline; PCR: polymerase chain reaction; PEtn: phosphorylethanolamine; PNA: peanut agglutinin; SDS-PAGE: sodium dodecyl suphate-polyacrylamide gel electrophoresis; TOCSY: total correlation spectroscopy.

\section{Acknowledgements}

This study was funded by an Australian Postgraduate Scholarship (to E.A.S.) and by a Griffith University grant (to A.P.M.) and fellowship (to C.J.D.). We thank Marcus Twomey and Jakob Rosenhauer for their contributions to purification of C. jejuni OS for the NMR studies.

\section{Author details}

IInstitute for Glycomics, Griffith University, Gold Coast campus, Queensland Australia. ${ }^{2}$ Microbiology, School of Natural Sciences, National University of Ireland, Galway, Ireland.

\section{Authors' contributions}

EAS carried out all of the electrophoretic and blotting experiments and drafted the initial manuscript. CJD aided with experimental work and participated in the design and coordination of the study and helped to draft the manuscript. IDG and JCW provided resources, aided in determination of the LOS structures with APM and helped draft the manuscript. APM and VK conceived this study, participated in its design, and the coordination and writing of the manuscript. All authors read and approved the final manuscript.

Received: 7 May 2010 Accepted: 30 November 2010 Published: 30 November 2010

\section{References}

1. Allos BM: Campylobacter jejuni Infections: update on emerging issues and trends. Clin Infect Dis 2001, 32(8):1201-1206.

2. Blaser MJ, Reller LB: Campylobacter enteritis. N Engl J Med 1981, 305(24):1444-1452 
3. Blaser MJ, Berkowitz ID, LaForce FM, Cravens J, Reller LB, Wang WL: Campylobacter enteritis: clinical and epidemiologic features. Ann Intern Med 1979, 91(2):179-185.

4. Godschalk PC, Kuijf ML, Li J, St Michael F, Ang CW, Jacobs BC, Karwaski MF, Brochu D, Moterassed A, Endtz HP, et al: Structural characterization of Campylobacter jejuni lipooligosaccharide outer cores associated with Guillain-Barre and Miller Fisher syndromes. Infect Immun 2007, 75(3):1245-1254

5. Nachamkin I, Allos BM, Ho T: Campylobacter species and Guillain-Barre syndrome. Clin Microbiol Rev 1998, 11(3):555-567.

6. Prendergast MM, Moran AP: Lipopolysaccharides in the development of the Guillain-Barre syndrome and Miller Fisher syndrome forms of acute inflammatory peripheral neuropathies. J Endotoxin Res 2000, 6(5):341-359.

7. Ang CW, Jacobs BC, Laman JD: The Guillain-Barre syndrome: a true case of molecular mimicry. Trends Immunol 2004, 25(2):61-66.

8. Ledeen RW, Yu RK: Gangliosides: structure, isolation, and analysis. Methods Enzymol 1982, 83:139-191.

9. Ropper AH: The Guillain-Barre syndrome. N Engl J Med 1992, 326(17):1130-1136

10. Gilbert M, Parker CT, Moran AP: Campylobacter jejuni lipooligosaccharides: structures and biosynthesis. In Campylobacter. 3 edition. Edited by: Nachamkin I, Szymanski CM, Blaser MJ. Washington, DC: ASM Press; 2008

11. Moran AP, Annuk H, Prendergast MM: Antibodies induced by gangliosidemimicking Campylobacter jejuni lipooligosaccharides recognise epitopes at the nodes of Ranvier. J Neuroimmunol 2005, 165(1-2):179-185.

12. Yuki N, Susuki K, Koga M, Nishimoto Y, Odaka M, Hirata K, Taguchi K, Miyatake T, Furukawa K, Kobata $T$, et al: Carbohydrate mimicry between human ganglioside GM1 and Campylobacter jejuni lipooligosaccharide causes Guillain-Barre syndrome. Proc Natl Acad Sci USA 2004, 101(31):11404-11409.

13. Blaser MJ, Hopkins JA, Berka RM, Vasil ML, Wang WL: Identification and characterization of Campylobacter jejuni outer membrane proteins. Infect Immun 1983, 42(1):276-284

14. Garenaux A, Jugiau F, Rama F, de Jonge R, Denis M, Federighi M, Ritz M: Survival of Campylobacter jejuni strains from different origins under oxidative stress conditions: effect of temperature. Curr Microbiol 2008, 56(4):293-297

15. Stintzi A: Gene expression profile of Campylobacter jejuni in response to growth temperature variation. J Bacteriol 2003, 185(6):2009-2016.

16. Parkhill J, Wren BW, Mungall K, Ketley JM, Churcher C, Basham D, Chillingworth T, Davies RM, Feltwell T, Holroyd S, et al: The genome sequence of the food-borne pathogen Campylobacter jejuni reveals hypervariable sequences. Nature 2000, 403(6770):665-668.

17. Gaynor EC, Cawthraw S, Manning G, MacKichan JK, Falkow S, Newell DG: The genome-sequenced variant of Campylobacter jejuni NCTC 11168 and the original clonal clinical isolate differ markedly in colonization, gene expression, and virulence-associated phenotypes. J Bacteriol 2004 186(2):503-517.

18. Karlyshev AV, Ketley JM, Wren BW: The Campylobacter jejuni glycome. FEMS microbiology reviews 2005, 29(2):377-390.

19. Moran AP, Zähringer U, Seydel U, Scholz D, Stütz P, Rietschel ET: Structural analysis of the lipid A component of Campylobacter jejuni CCUG 10936 (serotype 0:2) lipopolysaccharide. Description of a lipid A containing a hybrid backbone of 2-amino-2-deoxy-D-glucose and 2,3-diamino-2,3dideoxy-D-glucose. Eur J Biochem 1991, 198(2):459-469.

20. Oldfield NJ, Moran AP, Millar LA, Prendergast MM, Ketley JM: Characterization of the Campylobacter jejuni heptosyltransferase II gene, waaF, provides genetic evidence that extracellular polysaccharide is lipid A core independent. J Bacteriol 2002, 184(8):2100-2107.

21. St Michael F, Szymanski CM, Li J, Chan KH, Khieu NH, Larocque S, Wakarchuk WW, Brisson JR, Monteiro MA: The structures of the lipooligosaccharide and capsule polysaccharide of Campylobacter jejuni genome sequenced strain NCTC 11168. Eur J Biochem 2002, 269(21):5119-5136

22. Gilbert M, Brisson JR, Karwaski MF, Michniewicz J, Cunningham AM, Wu Y, Young NM, Wakarchuk WW: Biosynthesis of ganglioside mimics in Campylobacter jejuni $\mathrm{OH} 4384$. Identification of the glycosyltransferase genes, enzymatic synthesis of model compounds, and characterization of nanomole amounts by $600-\mathrm{mhz}{ }^{1} \mathrm{H}$ and ${ }^{13} \mathrm{C}$ NMR analysis. J Biol Chem 2000, 275(6):3896-3906
23. Linton D, Gilbert M, Hitchen PG, Dell A, Morris HR, Wakarchuk WW, Gregson NA, Wren BW: Phase variation of a beta-1,3 galactosyltransferase involved in generation of the ganglioside GM1-like lipo-oligosaccharide of Campylobacter jejuni. Mol Microbiol 2000, 37(3):501-514.

24. Peak IR, Grice ID, Faglin I, Klipic Z, Collins PM, van Schendel L, Hitchen PG, Morris HR, Dell A, Wilson JC: Towards understanding the functional role of the glycosyltransferases involved in the biosynthesis of Moraxella catarrhalis lipooligosaccharide. FEBS J 2007, 274(8):2024-2037.

25. Kuziemko GM, Stroh M, Stevens RC: Cholera toxin binding affinity and specificity for gangliosides determined by surface plasmon resonance. Biochemistry 1996, 35(20):6375-6384

26. Corcoran AT, Moran AP: Influence of growth conditions on diverse polysaccharide production by Campylobacter jejuni. FEMS Immunol Med Microbiol 2007, 49(1):124-132.

27. van der Woude MW, Baumler AJ: Phase and antigenic variation in bacteria. Clin Microbiol Rev 2004, 17(3):581-611.

28. Lipsitch $M, O^{\prime}$ Hagan JJ: Patterns of antigenic diversity and the mechanisms that maintain them. J R Soc Interface 2007, 4(16):787-802

29. Guerry P, Szymanski CM, Prendergast MM, Hickey TE, Ewing CP, Pattarini DL, Moran AP: Phase variation of Campylobacter jejuni 81-176 lipooligosaccharide affects ganglioside mimicry and invasiveness in vitro. Infection and immunity 2002, 70(2):787-793.

30. Bacon DJ, Szymanski CM, Burr DH, Silver RP, Alm RA, Guerry P: A phasevariable capsule is involved in virulence of Campylobacter jejuni 81-176. Mol Microbiol 2001, 40(3):769-777.

31. Hanniffy OM, Shashkov AS, Moran AP, Senchenkova SN, Savage AV: Chemical structure of the core oligosaccharide of aerotolerant Campylobacter jejuni O:2 lipopolysaccharide. Carbohydr Res 2001, 330(2):223-229.

32. Parker CT, Quinones B, Miller WG, Horn ST, Mandrell RE: Comparative genomic analysis of Campylobacter jejuni strains reveals diversity due to genomic elements similar to those present in C. jejuni strain RM1221. J Clin Microbiol 2006, 44(11):4125-4135.

33. Hitchcock PJ, Brown TM: Morphological heterogeneity among Salmonella lipopolysaccharide chemotypes in silver-stained polyacrylamide gels. $J$ Bacteriol 1983, 154(1):269-277.

34. Westphal O, Luderitz O, Bister F: Uber die Extraktion von Bakterien mit Phenol/Wasser. Naturforsch 1952, 7(b):148-155.

35. Chester IR, Murray RG: Analysis of the cell wall and lipopolysaccharide of Spirillum serpens. J Bacteriol 1975, 124(3):1168-1176.

36. Schagger H: Tricine-SDS-PAGE. Nat Protoc 2006, 1(1):16-22

37. Tsai CM, Frasch CE: A sensitive silver stain for detecting lipopolysaccharides in polyacrylamide gels. Anal Biochem 1982, 119(1):115-119.

38. Karlyshev AV, McCrossan MV, Wren BW: Demonstration of polysaccharide capsule in Campylobacter jejuni using electron microscopy. Infect Immun 2001, 69(9):5921-5924.

39. Laemmli UK: Cleavage of structural proteins during the assembly of the head of bacteriophage T4. Nature 1970, 227(5259):680-685.

40. Appelmelk BJ, Shiberu B, Trinks C, Tapsi N, Zheng PY, Verboom T, Maaskant J, Hokke CH, Schiphorst WE, Blanchard D, et al: Phase variation in Helicobacter pylori lipopolysaccharide. Infect Immun 1998, 66(1):70-76.

doi:10.1186/1471-2180-10-305

Cite this article as: Semchenko et al:: Temperature-dependent

phenotypic variation of Campylobacter jejuni lipooligosaccharides. BMC Microbiology 2010 10:305. 